\title{
Notre objectif commun: évaluer correctement les prestations médicales
}

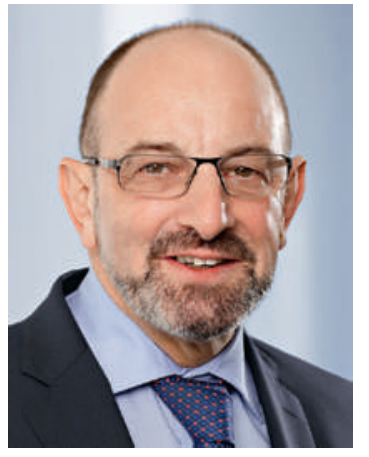

L'intervention tarifaire du Conseil fédéral sera bientôt réalité: l'ordonnance publiée le 20 juin dernier doit - conformément à la volonté de l'OFSP - être intégrée dans la structure tarifaire TARMED d'ici au $1^{\text {er }}$ octobre 2014. Les partenaires tarifaires curafutura, la FMH, H+, la CTM et santésuisse s'emploient donc dans l'urgence à mettre en place ses aspects techniques. Or rapidement, il s'est avéré que l'ordonnance présentait certaines lacunes, notamment sur des points techniques très concrets de sa mise en application, et que des interventions isolées dans la structure tarifaire complexe du TARMED étaient délicates et pouvaient avoir des effets négatifs sur l'ensemble de la structure.

Selon le texte de l'ordonnance, la nouvelle prestation 00.0015 ne peut être facturée qu'en lien avec les prestations de la médecine de famille. Mais le terme «prestations relevant de la médecine de famille» n'existe pas dans le TARMED actuel. Comment dès lors distinguer ces prestations des autres prestations du TARMED? Jusqu'ici, la Confédération n'a pas apporté de réponse à cette question. Même si cette position, qui vise à revaloriser la médecine de famille, n'est instaurée qu'à titre transitoire d'ici à l'achèvement de la révision globale du TARMED, elle conduira néanmoins à des pertes financières

\section{L'intervention tarifaire isolée du Conseil}

fédéral créera des incitatifs erronés. Seule une révision globale du TARMED conduira à une structure tarifaire appropriée.

pour les médecins de famille liées à l'effet de l'ordonnance sur d'autres positions. Nous sommes donc en droit de nous demander si les médecins de premier recours bénéficieront vraiment de l'amélioration promise par la Confédération et ce de manière durable.

La deuxième critique d'ordre général qu'il faut mentionner concerne le caractère approprié du contre-financement au moyen d'une réduction linéaire de 8,5\% de la prestation technique dans certains chapitres, qui créera immanquablement de nouveaux déséquilibres dans la structure tarifaire. Avec pour conséquence de nouvelles inégalités et des incitatifs erronés qui auront des effets au quotidien pour les médecins.
A cet égard, la Confédération n'a malheureusement pas tenu compte des points importants soulevés par les partenaires tarifaires lors de l'audition alors que les connaissances des partenaires sont indispensables pour garantir de bonnes solutions en matière de tarif.

L'intervention tarifaire du Conseil fédéral montre bien que seule une révision globale de la structure tarifaire du TARMED peut mener à une évaluation correcte des prestations et

L'OFSP veut la neutralité des coûts alors que la LAMal exige une structure tarifaire appropriée et conforme aux règles de l'économie d'entreprise.

des activités de toutes les spécialisations médicales. Heureusement, les travaux des partenaires impliqués, qui tiennent compte de leur propre savoir-faire technique et médical et bien entendu de celui des sociétés médicales, avancent bien.

Hormis la révision globale de la structure tarifaire conformément à la feuille de route et la création d'une nouvelle organisation TARMED Suisse sous la forme d'une société anonyme avec prise de décision à la majorité, il s'agit aussi de se préparer aux négociations concernant les prix. Selon le vice-directeur de l'OFSP, Oliver Peters, la structure devra être neutre en termes de points tarifaires, autrement dit, le nombre de points devra être le même avant et après la révision. Or la LAMal exige que les prestations soient fixées d'après les règles applicables en économie d'entreprise et structurées de manière appropriée. santésuisse souhaite même que les fournisseurs de prestations signent une convention de gestion des coûts alors que le contenu de la révision n'est pas encore connu. Cela reviendrait à instaurer un double plafonnement et une budgétisation globale, ce que la loi ne prévoit pas pour un tarif à la prestation national comme le TARMED.

Même si la séparation entre structure et prix est d'une importance capitale, nous devons commencer sans attendre à préparer les négociations. Nous avons besoin de nouvelles conventions, adaptées à la nouvelle situation tarifaire, qui puissent rapidement faire l'objet d'une négociation. Pour cela, les différentes organisations du corps médical doivent être unies et défendre ensemble leurs positions, selon le principe «Ensemble, nous sommes plus forts».

Dr Ernst Gähler, vice-président de la FMH, responsable du département Tarifs et conventions pour la médecine ambulatoire en Suisse / Professions paramédicales 